\title{
THE X-RAY SOURCES SCO X-1 AND CYG X-2 AS BINARIES
}

\author{
V. M. LYUTY
}

Sternberg Astronomical Institute, Moscow University, Moscow, U.S.S.R.

\section{(Abstract)}

\section{Sco X-1}

The analysis of photometric observations of the X-ray source Sco X-1 leads to the conclusion of the existence of a periodic component in the light variations of this source. For 1966-74, the results can be presented as

$$
\operatorname{Min}=\mathrm{JD} 2439946.58+3.9309 \mathrm{E} .
$$

The photoelectric observations of some authors are shown in Figure 1, using the 3.9309 period. Figure 2 shows the mean light-curve of Sco X-1. The amplitude $\Delta B$ is about $0^{m} \cdot 25$.

The data on radial velocities also confirm the existence of the 3.9309-day period. So Sco X-1 is a binary. The recent data on radial velocities obtained by Esipov from observations during 1971-72 are a strong confirmation of this conclusion.

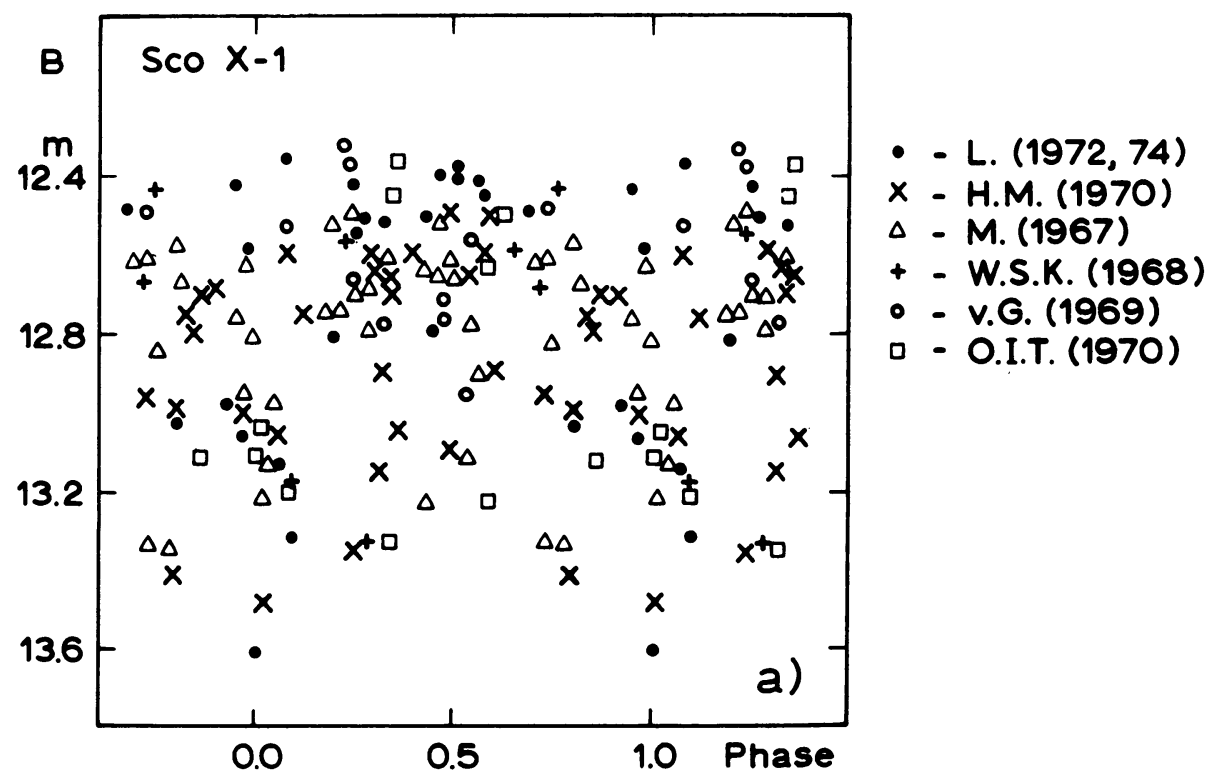

Fig. 1. Sco X-1 Min. =JD 2439946.58+3.9309 E, photoelectric observations: 125 nights, 1966-1974. 


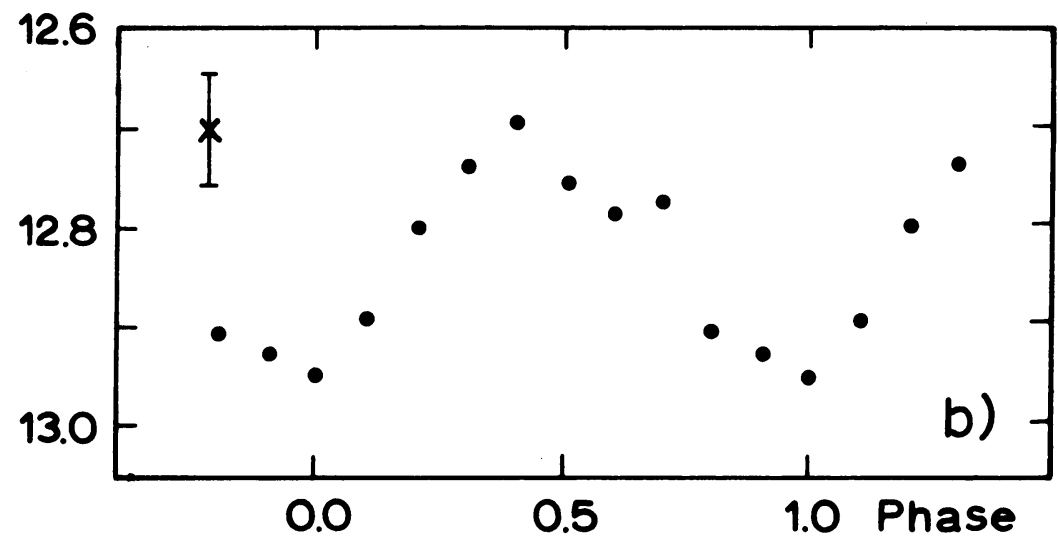

Fig. 2. Sco X-1 mean light curve.

\section{Cyg X-2}

All the available published data on radial velocities suggest the existence of a 0.251451 day period for this source. The orbital velocity is $180-200 \mathrm{~km} \mathrm{~s}^{-1}$, the mean velocity of the system is about $200 \mathrm{~km} \mathrm{~s}^{-1}$. The emission line He II 4686 velocity curve is in antiphase to the one from the absorption hydrogen lines (Figure 3).

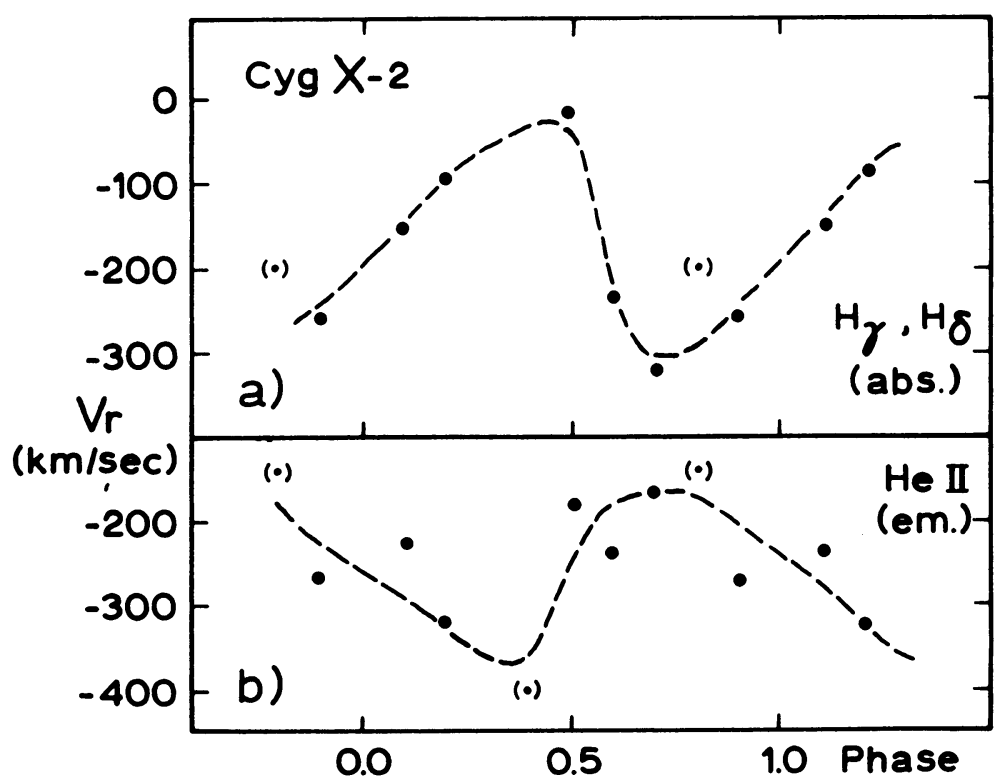

Fig. 3. Cyg X-2 mean velocity curves $(p=0.251451)$ - (a) absorption $\mathrm{H} \gamma, \mathrm{H} \delta$. (b) emission He II $\lambda 4686$. 